Rong Liu • Qiusheng Liu

\title{
Linear stability analysis of convection in two-layer system with an evaporating vapor-liquid interface*
}

Received: 21 January 2005 / Revised: 16 November 2005 / Accepted: 5 January 2006 / Published online: 13 April 2006

(c) Springer-Verlag 2006

\begin{abstract}
Classical theories have successfully provided an explanation for convection in a liquid layer heated from below without evaporation. However, these theories are inadequate to account for the convective instabilities in an evaporating liquid layer, especially in the case when it is cooled from below. In the present paper, we study the onset of Marangoni convection in a liquid layer being overlain by a vapor layer. A new two-sided model is put forward instead of the one-sided model in previous studies. Marangoni-Bénard instabilities in evaporating liquid thin layers are investigated with a linear instability analysis. We define a new evaporation Biot number, which is different from that in previous studies and discuss the influences of reference evaporating velocity and evaporation Biot number on the vapor-liquid system. At the end, we explain why the instability occurs even when an evaporating liquid layer is cooled from below.
\end{abstract}

Keywords Evaporation - Marangoni-Bénard instability · Convection

\section{Introduction}

The first systematic study of the convective pattern in a thin liquid layer was made by Bénard [1]. Rayleigh [2] developed a theory to explain the results of Bénard's experiments. The convection observed in Bénard's experiments was believed to be driven by buoyancy according to Rayleigh's theory. Nowadays this kind of buoyancy-driven convection is referred to as Rayleigh-Bénard convection. In Bénard's experiment, the liquid layer is so thin that the buoyancy effect is too weak to initiate the convection. Therefore, Rayleigh's theory is

\footnotetext{
* The project supported by the National Natural Science Foundation of China (10372105) and the Knowledge Innovation Program of Chinese Academy of Sciences (KJCX2-SW-L05 and KGCX-SW-409) The English text was polished by Keren Wang.
}

R. Liu · Q. S. Liu ( $\varangle)$

Institute of Mechanics, Chinese Academy of Sciences,

Beijing 100080, China

E-mail: liu@imech.ac.cn unsuitable for the convections in Bénard's experiment. In 1956, Block [3] introduced a new theory to explain the convections observed in Bénard's experiment. He demonstrated that the convection cells in a thin liquid layer are driven by variations in surface tension instead of buoyancy. Pearson [4] proposed a new theoretical model and demonstrated analytically that Bénard convection can be driven by variations in surface tension. This surface-tension-driven convection is referred as Marangoni-Bénard convection. Nield [5] found that buoyancy and surface tension are tightly coupled in Bénard's experiment. Pearson's and Nield's theories have successfully explained the onset of convection in a thin liquid layer without an evaporation interface. According to these theories, convective instability occurs only when the temperature gradient in the liquid is negative, i.e. the liquid is heated from below. In Block's qualitative description of the convection, the evaporating liquid layers were overlooked. Up to now, these theories still could not explain completely the convection in an evaporating liquid layer, especially in an evaporating liquid layer cooled from below. The convection instability induced by the coupling of evaporation and Marangoni effect in thin-liquid layers is not well explored. But a number of the industrial applications such as thin-film evaporators, boiling technologies and heat pipes involve evaporation processes, therefore, evaporative convection and instability may hold both theoretical and technological interests.

Various possible mechanisms, such as vapor recoil effect and nonlinear mass transfer effect, were suggested to explain instabilities in evaporation layers. Palmer [6] investigated the hydrodynamic stability of the rapidly evaporating liquids at a reduced pressure. Prosperetti [7] et al. proposed a physical model with a liquid layer of infinite depth undergoing steady evaporation. In his model, influence of viscosity is neglected and the destabilizing mechanisms only operative at short wavelength do not work. Bruelbach and Bankoff [8] considered a horizontal static liquid 1ayer which is evaporating when the plates are heated or condensing when the plates are cooled and analyzed its instabilities. In his model, vapor recoil, thermocapillary and rupture instabilities are discussed. Colinet and Legros [9] considered an evaporating liquid layer 


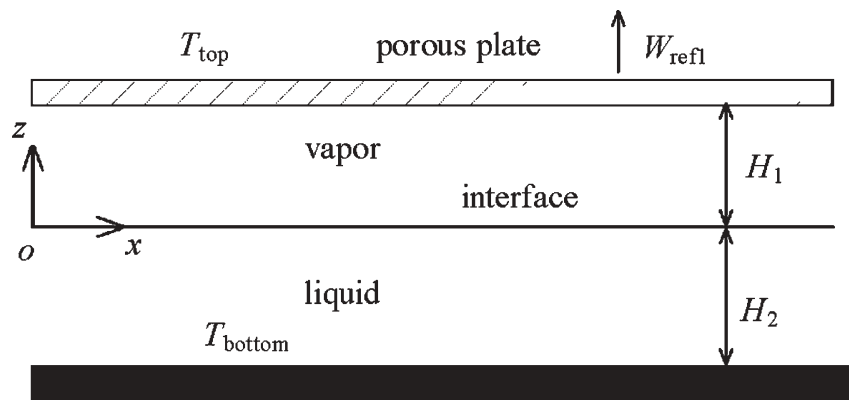

Fig. 1 Schematic diagram of the liquid-vapor layer system of definite depth

in contact with its own vapor and examined the possibility of the thermocapillary instabilities. In this model, dynamics of the vapor layer is decoupled from that of the liquid layer. The influence of evaporation only changes the boundary conditions in consequence. Since the dynamical coupling between liquid and vapor is not really considered in one-sided models mentioned above, they can not explain the instabilities in evaporating liquid layers.

Zhang and Chao $[10,11]$ conducted a systematic experimental investigation on fluid motions in evaporating liquid layers. The evaporation rate and the depth of the liquid have been found to be important parameters of the instability in an evaporating liquid layer. They proposed a new mechanism to explain the convection instabilities in an evaporating liquid layer. Modified forms of Marangoni number and Rayleigh number were used to describe stability status of evaporating layers. They pointed out that the modified form of Marangoni number is more suitable for describing the stability status of the liquid layer, but the convection as it is cooled from below was not considered in their work.

\section{Problem formulation}

In the present paper, we discuss the physical process of the evaporation in a vapor-liquid system. In our investigations, the dynamics of vapor is taken into account. A new theoretical two-sided model with an evaporating interface instead of one-sided one is proposed.

We examine the stability of a liquid-vapor system subjected to a vertical temperature gradient. The physical model is depicted in Fig. 1. A liquid layer of depth $\mathrm{H}_{2}$ is covered by its own vapor of depth $H_{1}$. The system is infinite in the horizontal direction and is finite in the vertical direction. The liquid layer is bounded from below by a rigid plate, and the vapor layer is bounded from above by a porous plate. The bottom and the top plates are considered as perfectly conducting boundaries. The temperature is $T_{\text {top }}$ at the top plate and $T_{\text {bottom }}$ at the bottom plate. The liquid-vapor interface is located at $z=0$. The gravitational acceleration, $g$, is in the negative $z$-direction. We assume that the velocity in $y$ direction is zero and all physical variables do not vary in $y$ direction. In this model, the vapor can be evacuated through the top porous plate by a pump that maintains a reduced pressure in the vapor layer. In reality, deformations will occurs as a result of hydrodynamic stresses caused by fluid motions. Here we assume that there is no deformation at the interface temporarily, and we will account for the deformation in future. The deformation concerns surface tension and gravity, and the conditions under which surface deformation is of second-order and can be safely neglected indeed exist. Colinet and Legros' analyses [9] indicate that in the limit $G a \rightarrow \infty$ and $C a \rightarrow 0$, the expression of the critical Marangoni number in the deformed interface system reduces to Pearson's relation where the interface is flat. $G a$ is the Galileo number defined as

$G a=\frac{\rho g d^{2}}{\sigma}$

and $C a$ is the capillary number defined as

$C a=\frac{\mu \kappa}{\sigma d}$,

where $\rho$ is the density of liquid; $g$ is the acceleration of gravity; $d$ is the thickness of the liquid layer; $\sigma$ is the surface tension; $\mu$ is the dynamic viscosity coefficient and $\kappa$ is the thermal diffusion coefficient of the liquid. Their theoretical results also indicate that the influence of deformations is effective only in the limit of long-wave, the effect of surface deformation quickly becomes negligible while increasing wavenumber $k$. For normal experimental situations, the Galileo number is very large while the capillary number is small, thus the effects of interface deformation are negligible. Hence, the evaporating interface may be assumed to be flat here.

Colinet and Legros [9] have considered a layer of evaporating pure liquid in contact with its own vapor of a depth of infinity, and examined the possibility of thermocapillary instability. The temperature in the vapor must not diverge when $z \rightarrow \infty$, which implies that the temperature is constant in the vapor. In this case, the vapor is like a passive gas and has little influence on the instability of the vapor liquid system. Introducing a coefficient, Biot number, to describe the heat exchange condition at the interface, the dynamics linking the liquid and vapor is decoupled, and the problem is reduced to Pearson's. In experiments, the temperature distribution is not as simple as that in Colinet's model and there is a nonuniform temperature distribution in the vapor layer.

In our model, the basic state is assumed to be one dimensional, i.e. all variables only depend on the vertical coordinate $z$, and the only non-zero component of the velocity in the vapor is the vertical one. We can imagine that there is an isothermal porous plate near the interface so as to introduce a temperature difference in the vapor layer instead of an isothermal vapor layer in Colinet's model. It is due to the porous plate that the level velocity is zero (non-skip condition) and the vertical one is non-zero(filtration condition). As discussed above, the two-sided model is more close to the real physical conditions than the one-sided model.

The interfacial tension satisfies a linear relation: $\sigma=$ $\sigma_{0}-\sigma_{T}\left(T-T_{0}\right)$, where $T_{0}$ is the reference temperature of 
interface and $-\sigma_{T}$ the surface tension variation per unit temperature.

Many investigators have studied the relations of the evaporation flux at the interface theoretically $[12,13]$ and experimentally $[14,15]$. In our model, we assume that the evaporating mass flux $J$ obeys the Hertz-Knudsen equation [16] at the interface:

$J=\beta \sqrt{\frac{M}{2 \pi R T}}\left(p_{s}(T)-p_{0}(T)\right)$,

where $\beta$ is the accommodation evaporation coefficient, $M$ is the molecular weight of vapor, $R$ is the universal gas constant, $p_{s}(T)$ is the saturation pressure at surface temperature $T, p_{0}(T)$ is the vapor pressure just beyond the interface. Equation (3) gives a general description of evaporation flux at the interface for a given local temperature. In the evaporation process, the pressure in vapor can be controlled by a pump, so the evaporating flux is a controllable physical parameter in our model.

\subsection{Dimensional controlling equations and boundary conditions}

For an incompressible viscid fluid, the equations governing the Marangoni convection of the liquid-vapor system with an evaporating interface are given as follows with Boussinesq approximation $[17,18]$ :

$$
\begin{aligned}
& \frac{\partial u_{i}}{\partial x}+\frac{\partial w_{i}}{\partial z}=0, \\
& \frac{\partial u_{i}}{\partial t}+u_{i} \frac{\partial u_{i}}{\partial x}+w_{i} \frac{\partial u_{i}}{\partial z} \\
& =-\frac{1}{\rho_{i}} \frac{\partial p_{i}}{\partial x}+v_{i} \nabla^{2} u_{i}, \frac{\partial w_{i}}{\partial t}+u_{i} \frac{\partial w_{i}}{\partial x}+w_{i} \frac{\partial w_{i}}{\partial z} \\
& =-\frac{1}{\rho_{i}} \frac{\partial p_{i}}{\partial z}+v_{i} \nabla^{2} w_{i}+\beta_{i} g\left(T_{i}-T_{0 i}\right), \\
& \frac{\partial T_{i}}{\partial t}+u_{i} \frac{\partial T_{i}}{\partial x}+w_{i} \frac{\partial T_{i}}{\partial z}=\kappa_{i} \nabla^{2} T_{i},
\end{aligned}
$$

where $u_{i}, w_{i}$ denote the horizontal velocity and vertical velocity, $p_{i}$ is the pressure, $T_{i}$ the temperature, and $T_{i 0}$ the reference temperature. The subscript $i(i=1,2)$ denotes the physical variables and properties of vapor or of liquid, respectively.

At the top plate, the horizontal velocity satisfies the nonslip condition, and the vertical velocity satisfies the filtration condition. The temperature there maintains a constant value. The boundary conditions are given as:

$z=H_{1}: u_{1}=0, \quad w_{1}=W_{\text {ref } 1}, \quad T_{1}=T_{\text {top }}$.

At the bottom plate, the velocity satisfies the nonslip condition and the temperature maintains a constant value, that is,

$z=-H_{2}: u_{2}=0, \quad w_{2}=0, \quad T_{2}=T_{\text {bottom }}$.

At the vapor-liquid interface $z=0$, and the interfacial mass balance equation is given by:

$J=\rho_{1}\left(w_{1}-w_{\text {int }}\right)=\rho_{2}\left(w_{2}-w_{\text {int }}\right)$, where $w_{\text {int }}$ is the velocity of the interface. The tangential components of velocities of liquid and vapor at the interface are continuous:

$u_{1}=u_{2}$.

We may assume that there is a thermodynamic equilibrium at the phase change interface, hence the temperature is continuous at the interface.

$T_{1}=T_{2}$.

The surface tension and its gradient with respect to the interfacial temperature are involved in the stress balance equation at the flat interface. By taking the dot product of the stress balance with the tangential and normal vectors, we will get the tangential and normal stress balance equations. The tangential stress equation at the interface is expressed by:

$\mu_{2} \frac{\partial u_{2}}{\partial z}-\mu_{1} \frac{\partial u_{1}}{\partial z}=\frac{\partial \sigma}{\partial x}$.

The normal stress equation at the interface is expressed by:

$$
\begin{gathered}
J\left(w_{2}-w_{1}\right)+\left(p_{2}-2 \mu_{2} \frac{\partial w_{2}}{\partial z}\right) \\
-\left(p_{1}-2 \mu_{1} \frac{\partial w_{1}}{\partial z}\right)=0 .
\end{gathered}
$$

The energy balance equation is given by

$$
\begin{aligned}
& J\left\{L+\frac{1}{2}\left(w_{2}-w_{\text {int }}\right)^{2}-\frac{1}{2}\left(w_{1}-w_{\text {int }}\right)^{2}\right\} \\
& \quad+\chi_{2} \frac{\partial T_{2}}{\partial z}-\chi_{1} \frac{\partial T_{1}}{\partial z}=0 .
\end{aligned}
$$

In addition, we need a relation connecting the mass flux $J$ to the local temperature, which is written as the linearized constitutive equation [8]:

$J=\frac{\beta \rho_{1} L}{T_{0}^{3 / 2}}\left(\frac{M}{2 \pi R}\right)^{1 / 2}\left(T_{\text {int }}-T_{0}\right)$,

where $L$ is the evaporation latent heat, $T_{\text {int }}$ the temperature of the liquid at the interface and $T_{0}$ the reference temperature.

\subsection{Dimensional perturbation equations and boundary conditions}

In unperturbed (base) state, we assume that there is no flow in the liquid layer and the velocity in the vapor layer is a constant, thus the components of the velocities in the base state are: $U_{\text {ref1 }}=0, W_{\text {ref1 }}=$ constant, $U_{\text {ref2 }}=0, W_{\text {ref2 }}=0$. If the evaporation rate is small, the temperature profile in the vapor layer is approximately linear.

To study the stability of the base state to small-amplitude perturbations, we follow the standard procedures of describing each flow variable as the sum of its base state value and a perturbation quantity. Here we will introduce two-dimensional small disturbances on the basic flow:

$\left(\begin{array}{c}u_{i} \\ w_{i} \\ T_{i} \\ p_{i}\end{array}\right)=\left(\begin{array}{c}U_{\mathrm{ref} i} \\ W_{\mathrm{ref} i} \\ T_{\mathrm{ref} i} \\ P_{\operatorname{ref} i}\end{array}\right)+\left(\begin{array}{c}u_{i}^{\prime} \\ w_{i}^{\prime} \\ T_{i}^{\prime} \\ p_{i}^{\prime}\end{array}\right)$. 
Substituting these expressions into the governing equations and boundary conditions, and retaining only the terms that are linear in the perturbation quantities, we obtain :

$$
\begin{aligned}
& \frac{\partial u_{i}^{\prime}}{\partial x}+\frac{\partial w_{i}^{\prime}}{\partial z}=0, \\
& \frac{\partial u_{i}^{\prime}}{\partial t}+W_{\operatorname{ref} i} \frac{\partial u_{i}^{\prime}}{\partial z}=-\frac{1}{\rho_{i}} \frac{\partial p_{i}^{\prime}}{\partial x}+v_{i} \nabla^{2} u_{i}^{\prime}, \\
& \frac{\partial w_{i}^{\prime}}{\partial t}+W_{\operatorname{ref} i} \frac{\partial w_{i}^{\prime}}{\partial z}=-\frac{1}{\rho_{i}} \frac{\partial p_{i}^{\prime}}{\partial z}+v_{i} \nabla^{2} w_{i}^{\prime}+\beta_{i} g T_{i}^{\prime}, \\
& \frac{\partial T_{i}^{\prime}}{\partial t}+W_{\operatorname{ref} i} \frac{\partial T_{i}^{\prime}}{\partial z}=\kappa_{i} \nabla^{2} T_{i}^{\prime} .
\end{aligned}
$$

It is possible to reduce the above set of equations to a simple one, by first taking $\partial / \partial x$ for Eq.(19) plus $\partial / \partial z$ Eq.(20) and using Eq.(18). We then get two equations for the pressure perturbations in vapor and liquid:

$\nabla^{2} p_{1}^{\prime}=\rho_{1} \beta_{1} \frac{\partial T_{1}^{\prime}}{\partial z}$,

$\nabla^{2} p_{2}^{\prime}=\rho_{2} \beta_{2} \frac{\partial T_{2}^{\prime}}{\partial z}$.

These allow eliminating $p_{1}^{\prime}, p_{2}^{\prime}$ as well as $u_{1}^{\prime}, u_{2}^{\prime}$ from this set of equations, by applying the operator $\nabla^{2}$ to Eq.(20). We then obtain:

$$
\begin{aligned}
& \frac{\partial}{\partial t} \nabla^{2} w_{1}^{\prime}+W_{\text {ref } 1} \frac{\partial}{\partial z} \nabla^{2} w_{1}^{\prime}=v_{1} \nabla^{2} \nabla^{2} w_{1}^{\prime}+\beta_{1} g \nabla_{k}^{2} T_{1}^{\prime}, \\
& \frac{\partial T_{1}^{\prime}}{\partial t}+\frac{\partial T_{\text {ref } 1}}{\partial z} w_{1}^{\prime}+W_{\text {ref } 1} \frac{\partial T_{1}^{\prime}}{\partial z}=\kappa_{1} \nabla^{2} T_{1}^{\prime}, \\
& \frac{\partial}{\partial t} \nabla^{2} w_{2}^{\prime}=v_{2} \nabla^{2} \nabla^{2} w_{2}^{\prime}+\beta_{2} g \nabla_{k}^{2} T_{2}^{\prime}, \\
& \frac{\partial T_{2}^{\prime}}{\partial t}+\frac{\partial T_{\text {ref } 2}}{\partial z} w_{2}^{\prime}=\kappa_{2} \nabla^{2} T_{2}^{\prime} .
\end{aligned}
$$

In this new set of equations only $w_{1}^{\prime}, w_{2}^{\prime}$ and $T_{1}^{\prime}, T_{2}^{\prime}$ are coupled, and Eqs.(24)-(27) are equivalent to Eqs.(18)-(21). They along with the corresponding boundary conditions are complete in the mathematical sense:

$$
\begin{aligned}
& \text { At } z=H_{1} \text { : } \\
& w_{1}^{\prime}=\frac{\partial w_{1}^{\prime}}{\partial z}=T_{1}^{\prime}=0, \\
& \text { At } z=H_{2} \text { : } \\
& w_{2}^{\prime}=\frac{\partial w_{2}^{\prime}}{\partial z}=T_{2}^{\prime}=0, \\
& \text { At } z=0 \text { : } \\
& \frac{\partial w_{1}^{\prime}}{\partial z}=\frac{\partial w_{2}^{\prime}}{\partial z}, \\
& T_{2}^{\prime}=T_{1}^{\prime} \text {, } \\
& \mu_{2}\left(\frac{\partial^{2} w_{2}^{\prime}}{\partial z^{2}}+\frac{\partial^{2} w_{2}^{\prime}}{\partial x^{2}}\right)-\mu_{1}\left(\frac{\partial^{2} w_{1}^{\prime}}{\partial z^{2}}+\frac{\partial^{2} w_{1}^{\prime}}{\partial x^{2}}\right) \\
& =-\sigma_{T} \frac{\partial^{2} T_{2}^{\prime}}{\partial x^{2}},
\end{aligned}
$$

$$
-J^{\prime} L+\chi_{1} \frac{\partial T_{1}^{\prime}}{\partial z}=\chi_{2} \frac{\partial T_{2}^{\prime}}{\partial z}
$$

$$
\frac{\rho_{1} \rho_{2}}{\rho_{2}-\rho_{1}}\left(w_{1}^{\prime}-w_{2}^{\prime}\right)=\frac{\beta \rho_{1} L}{T_{0}^{3 / 2}} \sqrt{\frac{M}{2 \pi R}} T_{2}^{\prime},
$$

in which the evaporation flux at the interface is:

$$
J^{\prime}=\frac{\rho_{1} \rho_{2}}{\rho_{2}-\rho_{1}}\left(w_{1}^{\prime}-w_{2}^{\prime}\right)
$$

With these equations and corresponding boundary conditions, we can solve the vertical velocities and temperatures in the liquid and vapor layers.

The dynamic boundary condition at the interface is:

$$
\begin{gathered}
2 J_{\text {ref }}\left(w_{2}^{\prime}-w_{1}^{\prime}\right)+\left(p_{2}^{\prime}-2 \mu_{2} \frac{\partial w_{2}^{\prime}}{\partial z}\right) \\
-\left(p_{1}^{\prime}-2 \mu_{1} \frac{\partial w_{1}^{\prime}}{\partial z}\right)=0 .
\end{gathered}
$$

Boundary condition (36) is decoupled from Eqs.(24)-(27) and boundary conditions (28)-(34). Since we only want to investigate the instability of the system and do not want the complete variables including the pressure $p_{1}$ and $p_{2}$, the dynamic boundary condition at the interface is not applied in our analyses.

\subsection{Scaled perturbation equations and boundary conditions}

We choose the depth of the liquid layer $\mathrm{H}_{2}$ as the length scale, $v_{2} / H_{2}$ as the velocity scale, $H_{2}^{2} / \nu_{2}$ as the time scale, the temperature difference $\Delta T=T_{\text {bottom }}-T_{\text {top }}$ as the temperature scale. The dimensionless ratio of thermal diffusive, thermal volumetric expansion coefficient, thermal conductivity, dynamic viscosity, density, kinematic viscosity are $\kappa^{*}=$ $\kappa_{1} / \kappa_{2}, \beta^{*}=\beta_{1} / \beta_{2}, \chi^{*}=\chi_{1} / \chi_{2}, \mu^{*}=\mu_{1} / \mu_{2}, \rho^{*}=$ $\rho_{1} / \rho_{2}, v^{*}=\nu_{1} / \nu_{2}$, respectively. In the next parts of the present paper, the scaled equations, along with the boundary conditions are used to analyze the stability of small perturbations. For the sake of simplicity, all the variables are scaled unless it is explicitly stated that they are dimensional.

In order to carry out the instability analysis on the liquidvapor system, the perturbations are then expanded in terms of normal modes, with the forms:

$$
\left(\begin{array}{c}
u_{i}^{\prime} \\
w_{i}^{\prime} \\
T_{i}^{\prime} \\
p_{i}^{\prime}
\end{array}\right)=\left(\begin{array}{c}
\tilde{U}_{i}(z) \\
\tilde{W}_{i}(z) \\
\tilde{\Theta}_{i}(z) \\
\tilde{P}_{i}(z)
\end{array}\right) \exp [\lambda t+\mathrm{i} k x],
$$

in which $\lambda$ is the time growth factor, $k$ the wavenumber, and $\tilde{U}_{i}(z), \tilde{W}_{i}(z), \tilde{P}_{i}(z), \tilde{\Theta}_{i}(z)$ are the amplitudes of horizontal velocity, vertical velocity, pressure and temperature, respectively.

Substituting these expansions into Eqs.(24)-(27) and boundary conditions (28)-(34), we have,

$$
\begin{gathered}
\nu^{*}\left(\mathrm{D}^{2}-k^{2}\right)^{2} \tilde{W}_{1}-W_{\text {ref } 1} \mathrm{D}\left(\mathrm{D}^{2}-k^{2}\right) \tilde{W}_{1} \\
-\beta^{*} G r k^{2} \tilde{\Theta}_{1}=\lambda\left(\mathrm{D}^{2}-k^{2}\right) \tilde{W}_{1},
\end{gathered}
$$




$$
\begin{aligned}
& \kappa^{*}\left(\mathrm{D}^{2}-k^{2}\right) \tilde{\Theta}_{1}-W_{\text {ref1 }} \operatorname{Pr} \mathrm{D} \tilde{\Theta}_{1}-\operatorname{Pr} \frac{\partial T_{\text {ref1 }}}{\partial z} \tilde{W}_{1} \\
& =\lambda \operatorname{Pr} \tilde{\Theta}_{1}, \\
& \left(\mathrm{D}^{2}-k^{2}\right)^{2} \tilde{W}_{2}-G r k^{2} \tilde{\Theta}_{1}=\lambda\left(\mathrm{D}^{2}-k^{2}\right) \tilde{W}_{2}, \\
& \left(\mathrm{D}^{2}-k^{2}\right) \tilde{\Theta}_{2}-\operatorname{Pr} \frac{\partial T_{\text {ref2 }}}{\partial z} \tilde{W}_{2}=\lambda \operatorname{Pr} \tilde{\Theta}_{2}, \\
& \tilde{W}_{1}=0, \quad \mathrm{D} \tilde{W}_{1}=0, \quad \tilde{\Theta}_{1}=0 \quad \text { at } z=h_{1}, \\
& \tilde{W}_{2}=0, \quad \mathrm{D} \tilde{W}_{2}=0, \quad \tilde{\Theta}_{2}=0 \quad \text { at } z=-1 . \\
& \text { At the interface } z=0: \\
& \mathrm{D} \tilde{W}_{1}=\mathrm{D} \tilde{W}_{2}, \quad \tilde{W}_{2}=\rho^{*} \tilde{W}_{1}, \quad \tilde{\Theta}_{2}=\tilde{\Theta}_{1}, \\
& \tilde{W}_{1}-\tilde{W}_{2}=M a \cdot E \cdot B i_{\mathrm{ev}} \cdot \tilde{\Theta}_{2}, \\
& \mathrm{D} \tilde{\Theta}_{2}-\chi^{*} \mathrm{D} \tilde{\Theta}_{1}+B i_{\mathrm{ev}} \cdot \tilde{\Theta}_{2}=0, \\
& \mu^{*}\left(\mathrm{D}^{2}+k^{2}\right) \tilde{W}_{1}-\left(\mathrm{D}^{2}+k^{2}\right) \tilde{W}_{2}=k^{2} \frac{M a}{P r} \tilde{\Theta}_{2},
\end{aligned}
$$

where $\mathrm{D}$ stands for the dimensionless differential operator with respect to the vertical coordinate $\mathrm{d} / \mathrm{d} z, \lambda$ the dimensionless time growth rate, and $k$ the dimensionless wavenumber. Dimensionless numbers include Grashof number defined as

$G r=\frac{g \beta_{2} H_{2}^{3} \Delta T}{v_{2}^{2}}$

Prandtl number defined as

$\operatorname{Pr}=\frac{\nu_{2}}{\kappa_{2}}$,

Marangoni number of the system defined as

$M a=\frac{\sigma_{T} \Delta T H_{2}}{\mu_{2} \kappa_{2}}$,

the dimensionless number $E$ defined as

$E=\frac{\kappa_{2} \chi_{2}\left(1-\rho^{*}\right)}{\rho^{*} \sigma_{T} L H_{2}}$

and the evaporating Biot number $B i_{\mathrm{ev}}$ defined as

$$
B i_{\mathrm{ev}}=\beta \rho_{1} L^{2} H_{2} \sqrt{M / 2 \pi R T_{0}^{3}} / \chi_{2} \text {. }
$$

Boundary condition (46) is obtained from the energy balance equation at the interface. In Eq.(46), the evaporation Biot number $B i_{\mathrm{ev}}$ gives the ratio between the evaporation latent heat at the interface and the conduction heat in the liquid. According to boundary condition (45), the evaporation Biot number is the ratio between $\mathcal{O}\left(\tilde{\Theta}_{2}\right)$ and $\mathcal{O}\left(\tilde{W}_{1}-\tilde{W}_{2}\right)$. Here $\mathcal{O}\left(\Theta_{2}\right)$ is the scale of the perturbation temperature at the interface, and $\mathcal{O}\left(\tilde{W}_{1}-\tilde{W}_{2}\right)$ is the scale of the perturbation velocity. When $B i_{\mathrm{ev}} \rightarrow 0$ and the temperature perturbation at the interface is large, the system becomes unstable; when $B i_{\mathrm{ev}} \rightarrow \infty$, and there is no temperature perturbation at the interface, the system is absolutely stable. The critical temperature difference of the system can be obtained according to Eq.(50). Here we define a similar dimensionless number, Marangoni number of the liquid layer

$M a_{2}=\frac{\sigma_{T} \Delta T_{2} H_{2}}{\mu_{2} \kappa_{2}}$,

in which $\Delta T_{2}=T_{\text {bottom }}-T_{\text {int }}, M a_{2}$ will be discussed when we consider the temperature difference $\left(\Delta T_{2}\right)$ in the liquid layer instead of that in the system $(\Delta T)$.

\section{Numerical method}

Spectral method belongs to the general class of weighted residual methods for which approximations are sought in terms of a truncated series expansion, such that some quantity (error or residual) which should be exactly zero is forced to be zero only in an approximate sense. This is done through the scalar product

$(u, v)_{w}=\int_{\alpha}^{\beta} u(x) v(x) w(x) \mathrm{d} x$,

where $u(x)$ and $v(x)$ are two functions defined on $[\alpha, \beta]$ and $w(x)$ is some given weight function.

Let us consider the solution of the differential equation

$\mathcal{L} u-f=0, \quad-1<x<1$,

where $\mathcal{L}$ is a differential operator assumed to be linear and of $P$ order. Along with Eq.(55), we have the following linear boundary conditions:

$\mathcal{B}_{i} u=g_{i}, \quad i=1, \ldots, P$,

where $\mathcal{B}_{i}$ is an operator determined by boundary conditions. The solution of Eq.(55) and boundary condition (56) is sought in the form

$u_{N}(x)=\sum_{k=0}^{N} \hat{u}_{k} \varphi_{k}(x)$,

in which $\varphi_{k}(x)$ is the $k$ th expansion function, and $\hat{u}_{k}$ is the $k$ th spectral coefficient.

There are at least two methods for us to solve the general eigenvalue problem. One is the collocation method and the other is the tau method. The so-called tau method is a modification of the Galerkin method allowing the use of trial functions not satisfying the homogeneous boundary conditions.

The equations determining the $N+1$ coefficients $\hat{u}_{k}$, $k=0, \ldots, N$ are obtained by considering the Galerkin equations with $R_{N}=\mathcal{L} u-f$ and $i=0, \ldots, N-P$, therefore,

$\sum_{k=0}^{N} \hat{u}_{k}\left(\mathcal{L} \varphi_{k}, \varphi_{i}\right)_{w}=\left(f, \varphi_{i}\right)_{w}, \quad i=0, \ldots, N-P$.

These equations are completed with the boundary conditions

$\mathcal{B}_{i} u_{N}=g_{i}, \quad i=1, \cdots, P$

Theoretical studies on the approximation error may be found in Ref. [19]. Error estimates might be obtained for functional spaces weighted with the Chebyshev weight. For example, the error of the Galerkin approximation, defined in the $H_{w}^{p}(-1,1)$-norm, is found to satisfy

$\left\|u-u_{N}\right\|_{H_{w}^{p}(-1,1)} \leq C N^{-1 / 2+2 p-m}|| u \|_{H_{w}^{m}}(-1,1)$,

for $1 \leq p \leq m$, if $u \in H_{w}^{m}(-1,1)$ for some $m \geq 1$. The constant $C$ is independent of $N$. The space $H_{w}^{p}(-1,1)$ is the 
weighted Sobolev space of order $p$, whose norm is defined by

$$
\|u\|_{H_{w}^{p}}(-1,1)=\left(\sum_{k=0}^{p} \int_{-1}^{1}\left|u^{(k)}(x)\right|^{2} w(x)\right)^{1 / 2} .
$$

The complete solution of the linear stability problem is determined once Eqs. (38)-(41) subject to the boundary conditions (42)-(44) are solved. In order to discretize these equations and boundary conditions in $[-1,1]$ and solve the general eigenvalue problem using spectral method(Tau-Chebyshev method) [20,21], we introduce these transformations:

$\eta_{1}=\frac{2}{h_{1}} z-1$,

where $z \in\left[0, h_{1}\right], \eta_{1} \in[-1,1]$,

$\eta_{2}=\frac{2}{h_{2}} z+1$,

where $z \in\left[-h_{2}, 0\right], \eta_{2} \in[-1,1]$.

Let $\varphi_{n}(x)=T_{n}(x)$, where $T_{n}(x)$ denotes the $n$ th-degree Chebyshev polynomial of the first kind, defined by

$T_{n}(x)=\cos (n \arccos x), \quad x \in[-1,1]$

for all non-negative integers $n$. It is possible to expand a function $F(x)$ in interval $-1 \leq x \leq 1$ as

$F(x)=\sum_{n=0}^{\infty} \hat{F}_{n} T_{n}(x)$

where $\hat{F}_{n}=\frac{2}{\pi c_{n}} \int_{-1}^{1} f(x) T_{n}(x)\left(1-x^{2}\right)^{-1 / 2} \mathrm{~d} x$, with $c_{0}=2$, $c_{n}=1(n>0)$. form

We seek an approximate solution of Eqs.(38)-(41) of the

$$
\begin{aligned}
& \tilde{W}_{1}(\eta)=\sum_{n=0}^{N} \hat{W}_{1 n} T_{n}(\eta), \\
& \tilde{W}_{2}(\eta)=\sum_{n=0}^{N} \hat{W}_{2 n} T_{n}(\eta), \\
& \tilde{\Theta}_{1}(\eta)=\sum_{n=0}^{N} \hat{\Theta}_{1 n} T_{n}(\eta), \\
& \tilde{\Theta}_{2}(\eta)=\sum_{n=0}^{N} \hat{\Theta}_{2 n} T_{n}(\eta) .
\end{aligned}
$$

The Chebyshev polynomial approximations used here are of infinite order in the sense that the error decreases more rapid than any power of $1 / N$ as $N \rightarrow \infty$. In our computation, a truncation of 21 terms $(N=20)$ is accurate enough.

Once tau method is selected to obtain equations for the expansion coefficients $\hat{W}_{i n}, \hat{\Theta}_{i n}(i=1,2)$, there remains the problem of solving the general eigenvalue problem.
Table 1 Physical properties of alcohol and alcohol vapor at $298 \mathrm{~K}$ in saturated state

$$
\begin{array}{ll}
\hline \rho_{1}=1.87 \times 10^{-1} \mathrm{~kg} / \mathrm{m}^{3} & \rho_{2}=0.804 \times 10^{3} \mathrm{~kg} / \mathrm{m}^{3} \\
\mu_{1}=8.65 \times 10^{-6} \mathrm{~Pa} \cdot \mathrm{s} & \mu_{2}=1.07 \times 10^{-3} \mathrm{~Pa} \cdot \mathrm{s} \\
\nu_{1}=4.63 \times 10^{-5} \mathrm{~m}^{2} / \mathrm{s} & \nu_{2}=1.33 \times 10^{-6} \mathrm{~m}^{2} / \mathrm{s} \\
\kappa_{1}=5.73 \times 10^{-5} \mathrm{~m}^{2} / \mathrm{s} & \kappa_{2}=9.36 \times 10^{-8} \mathrm{~m}^{2} / \mathrm{s} \\
\beta_{1}=3.35 \times 10^{-3} \mathrm{~K}^{-1} & \beta_{2}=3.02 \times 10^{-4} \mathrm{~K}^{-1} \\
\chi_{1}=1.53 \times 10^{-2} \mathrm{~W} /(\mathrm{m} \cdot \mathrm{K}) & \chi_{2}=1.79 \times 10^{-1} \mathrm{~W} /(\mathrm{m} \cdot \mathrm{K}) \\
L=9.19 \times 10^{2} \mathrm{~kJ} / \mathrm{kg} & \\
\sigma=23.5 \times 10^{-3} \mathrm{~N} / \mathrm{m} & \\
\sigma_{T}=-8.32 \times 10^{-5} \mathrm{~N} /(\mathrm{m} \cdot \mathrm{K}) & \\
\hline
\end{array}
$$

$$
\boldsymbol{A} \boldsymbol{X}=\lambda \boldsymbol{B} \boldsymbol{X}
$$

where $\boldsymbol{A}$ and $\boldsymbol{B}$ are two matrices of dimensions $4(N+1) \times$ $4(N+1), \boldsymbol{X}$ is a combination of the spectral coefficients of $\hat{W}_{1}(\eta), \hat{W}_{2}(\eta), \hat{\Theta}_{1}(\eta), \hat{\Theta}_{2}(\eta)$ of dimension $4(N+1)$.

The eigenvalue $\lambda=0$ corresponds the onset of steady convection. The marginal stability curves in the $(k, M a)$ plane where $\operatorname{Re}(\lambda)=0$ separate regions of unstable modes with $\operatorname{Re}(\lambda)>0$ from regions of stable modes with $\operatorname{Re}(\lambda)<$ 0 .

\section{Discussion of results}

The calculations are carried out, using the physical properties of alcohol and alcohol vapor at its saturation temperature $T_{s}=298 \mathrm{~K}$. The depth of the liquid layer is $1 \mathrm{~mm}$ and the depth ratio between the vapor layer and liquid layer is $h=1$. The ratios of physical properties (see Ref. [22]) and dimensionless numbers of the vapor-liquid system are $v^{*}=34.7$, $\rho^{*}=2.3 \times 10^{-4}, \chi^{*}=8.5 \times 10^{-2}, \kappa^{*}=6.1 \times 10^{2}$, $P r=14.9$ and $G r=0$ (microgravity condition), respectively.

The evaporation is rather complicated, and it is helpful to study different aspects of its influences on the system separately whenever possible. The evaporation Biot number and the evaporating velocity are two parameters responsible for the instabilities of the system. The Biot number is related to the influence of the evaporation on the interface heat exchange, whereas the evaporating velocity influences the temperature distribution in the unperturbed liquid layer and vapor layer. The evaporation Biot number is close to zero as $\beta \rightarrow 0$. From Eq.(3), we obtain

$0 \leq W_{\text {ref1 }} \leq 91.0 \beta$

for alcohol. For a given $\beta$, the evaporation velocity is not an arbitrary parameter and varies only in a limited range according to Eq.(69). No matter how small $\beta$ is, the velocity is not necessarily very small. For example, when $\beta=0.001$, the evaporation velocity can reach $0.0091 \mathrm{~m} / \mathrm{s}$. In experiment, the evaporation velocity is about $0.1 \mathrm{~mm} / \mathrm{s}$ or even smaller. As discussed above, even though $\beta$ is close to zero, we can still get an evaporating velocity not close to zero. In some sense, the evaporation accommodation coefficient is free from the limitation of evaporating velocity and vice versa, except in 


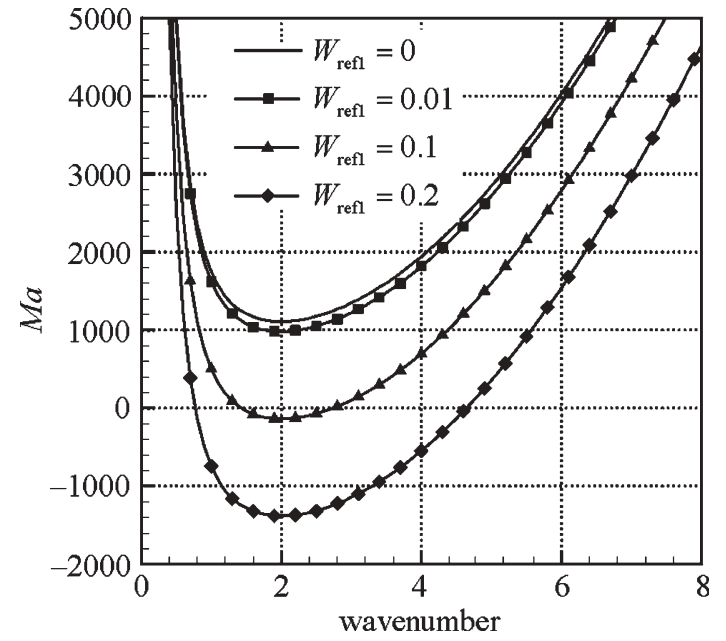

Fig. 2 Marangoni number versus dimensionless wavenumber for different evaporation velocities in a definite depth $\operatorname{system}\left(G r=0, B i_{\mathrm{ev}}=0\right)$

the case when $\beta$ is too small. Since there is little difference between zero Biot number and a very small Biot number for the instability of the system, we extend our computation to zero Biot number with a non-zero evaporating velocity. The case that $B i_{\mathrm{ev}}=0$ and $W_{\mathrm{ref} 1}>0$ can be envisioned as a physical limit of very weak evaporating ability, whereas a relatively greater pressure difference drives a greater evaporating flux at the interface.

We wish to examine how the evaporation influences the instability of the liquid-vapor system of definite depth. From our numerical results, the evaporation influences the convection instability of the system through two ways: the reference evaporation velocity influences the stability of system by changing the reference temperature distribution of the liquid layer; the evaporation Biot number, which is related to the degree of temperature perturbation at the interface, influences the stability of the system through boundary conditions.

\subsection{Influence of reference evaporation velocity on the critical values (when $B i_{\mathrm{ev}}=0$ )}

When $B i_{\mathrm{ev}}=0$, there is no perturbation of evaporation velocity. If the evaporating velocity is zero, the instability analysis gives the classic Marangoni-Bénard convection in a two-layer system without evaporation as described in Refs. $[23,24]$.

The curves in Fig. 2 represent the loci of neutrally stable perturbations in a liquid-vapor system of definite depth. At a given evaporating velocity, the base state is stable against disturbances with a given wavenumber $k$, provided that the Marangoni number is below the value given in Fig. 2. Once the Marangoni number exceeds this threshold, the perturbation with wavenumber $k$ has a positive growth rate. In Fig. 2, the smallest Marangoni number in each locus corresponds the critical Marangoni number of the system.

In Fig. 2, the greater the evaporation velocity is, the smaller the critical Marangoni number of the system is, i.e.,the liquid

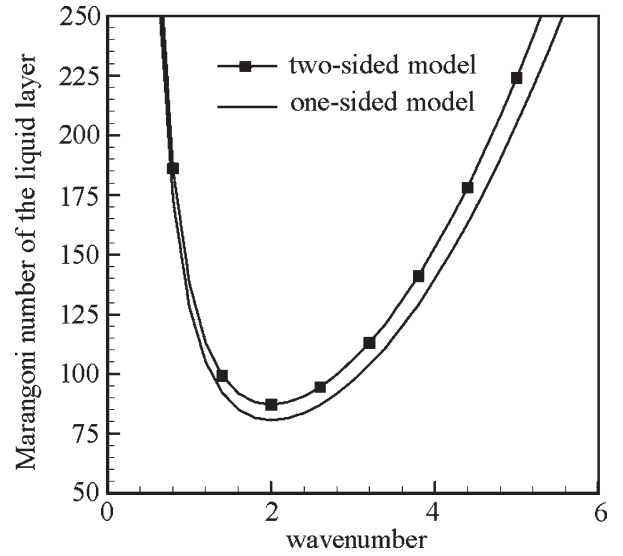

Fig. 3 Comparison between the relation of Marangoni number of the liquid layer versus dimensionless wavenumber in two-sided model and that in one-sided model $(B i=0$ in the curve of the one-sided model, $B i_{\mathrm{ev}}=0$ in the curve of the two-sided model)

layer becomes more stable with the decrease of the evaporating velocity. The locus with evaporation velocity $W_{\text {ref1 }}=0$ is the most stable in Fig. 2.

When the evaporating velocity is greater than a certain value, for Example, $W_{\text {ref1 }} \geq 0.1$, the critical Marangoni number is less than zero. In this case, the system is instable even though it is cooled from below. This phenomenon could not be found in previous one-sided models. In a classical one-sided Marangoni-Bénard model, Marangoni-Bénard convection is established only when the temperature gradient is negative, i.e., the convection occurs only when the layer is heated from below according to classical theories. In our two-sided model, the temperature distribution of the reference state plays a major role in the stability of the system.

From $M a$, we can only get the temperature difference between the top and the bottom plates. It is necessary to introduce $M a_{2}$ in order to get the temperature difference in the liquid layer. Note that the actual value of the liquid layer Marangoni number $M a_{2}$ defined in Eq.(53) is in direct proportion to the evaporation-driven temperature difference $\Delta T_{2}$, which can be calculated according to Eq.(15). For a given temperature $T_{\text {bottom }}$ and $T_{\text {top }}$, with the vapor pressure $p_{1}$ as the control parameter, the evaporating velocity is proportional to $p_{s}-p_{1}$ according to the Hertz Knudsen equation (Eq.(3)). So when we change the pressure of the vapor layer, the temperature distribution will be changed with respect to the evaporating velocity.

Figure 3 shows the Marangoni number of the liquid layer $M a_{2}$ versus wavenumber. The relation linking the temperature difference of the two layers and that of the liquid layer in the definite depth liquid-vapor system is:

$\Delta T_{2}=\left(J L H_{1}+\chi_{1} \Delta T\right) H_{2} /\left(\chi_{2} H_{1}+\chi_{1} H_{2}\right)$.

From Eq.(70), even the liquid-vapor system is cooled from below ( $\Delta T<0$ and $M a<0$ in Fig. 2), the temperature difference in the liquid layer can be positive $\left(\Delta T_{2}>0\right.$ and $M a_{2}>0$ in Fig. 3), because the value of $J L H_{1}$ is positive. In Fig. 3, the corresponding critical Marangoni numbers of the 


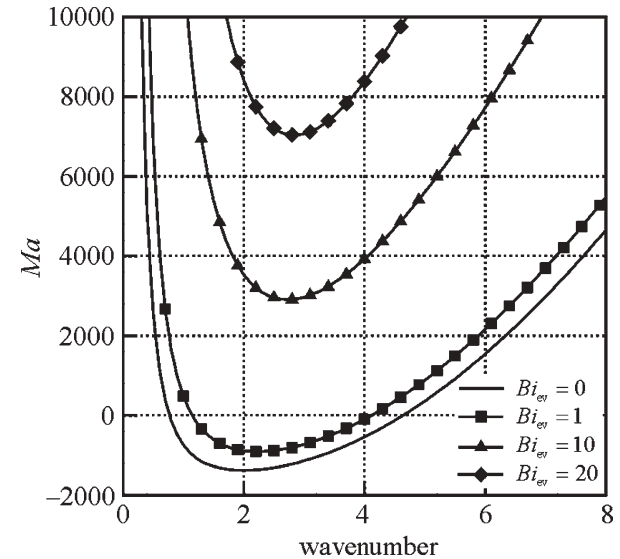

Fig. 4 Marangoni number versus dimensionless wavenumber for different evaporation Biot numbers in a definite depth $\operatorname{system}(\mathrm{Gr}=$ $\left.0, W_{\text {ref1 }}=0.2\right)$

liquid layer, $M a_{2}$, for different evaporation velocities are all positive. It is notable that no matter what value the evaporation velocity is, the convection occurs as long as $M a_{2}$ exceeds a certain value. Since $M a_{2}$ is independent of the evaporation rate, the curve of $M a_{2}$ for different evaporation rates is the same. From the discussion above, even the liquid layer is cooled from below, the temperature difference in the liquid layer can be negative for the cooling effect of evaporation at the interface.

In Fig. 3, the continuous line locus is the result of classical one-sided model. In one-sided model, the dynamics of the vapor is overlooked. In our two-sided model, the Maragoni number of the liquid layer is greater than that in one-sided model for all wavenumbers. This means that the vapor layer has a stable effect on the system while only considering the dynamics effect of the vapor layer on the liquid layer.

\subsection{Influence of evaporation Biot number on the critical values (when $B i_{\mathrm{ev}} \neq 0$ )}

Figure 4 shows the neutral stability curves with different evaporating Biot numbers. The evaporating Biot number represents the capability of heat exchange by evaporating at the liquid interface. If there is no evaporation velocity perturbation, the correspondent Biot number is zero. When the evaporating accommodation coefficient or the evaporating latent heat of the vapor is close to zero, the instability status of the system is close to that of a system with zero Biot number. When $W_{\text {ref1 }}=0.2$ and $B i_{\mathrm{ev}}=0$, the Maragoni number is negative. The critical Marangoni numbers increases with the evaporation Biot number. When $B i_{\mathrm{ev}}$ exceeds a certain value, the Marangoni number becomes positive. This means that the vapor-liquid system becomes more and more stable with the increase of the Biot number.

Table 2 gives the critical Marangoni number of the liquid layer for different evaporating Biot numbers. The liquid Marangoni number also increases with the evaporating Biot number. The Marangoni number is always positive even when
Table 2 Critical Marangoni number of the liquid layer versus different evaporation Biot numbers in a definite depth $\operatorname{system}\left(G r=0, W_{\text {ref1 }}=\right.$ $0.2, h=1)$

\begin{tabular}{llllll}
\hline$B i_{\mathrm{ev}}$ & 0 & 1 & 5 & 10 & 20 \\
$M a_{2}$ & 86.6 & 123.7 & 260.1 & 424.7 & 749.4 \\
\hline
\end{tabular}

the Marangoni number of the system is negative, for example, when $B i_{\mathrm{ev}}=0, W_{\text {ref1 }}=0.2$.

In classical theories, the Biot number is introduced into the boundary conditions of thermal equilibrium, i.e., $\mathrm{d} T / \mathrm{d} z+$ $B i T=0$, in which $B i$ is defined as $q d / \chi, q$ is the Newton cooling coefficient, $d$ the depth of the liquid layer, and $\chi$ the thermal conductivity coefficient. The problem of a liquid cooling by evaporation may be treated in this fashion. In this case, the one-sided model can be used, for which the dynamics of the vapor phase can be decoupled from that of the liquid phase. The effect of evaporation may be reasonably represented by a given heat loss from the surface. The Biot number is considered to depend on the rate of evaporation and the latent heat, and the stronger the evaporation is, the larger the Biot number will be. But according to classical results, the system becomes more and more stable with the increase of the Biot number. This result is obviously inconsistent with the observations in experiment because the evaporating liquid layer is more unstable than a non-evaporating one in experiments and convection cells in evaporating layers are observed even with a lower critical Maranogni number.

In our two-sided model, the evaporation Biot number depends on the perturbation evaporation rate, i.e., the accommodation evaporation coefficient and latent heat, instead of the reference evaporation rate. $B i_{\mathrm{ev}}=0$ or $\infty$ presents a different special case, respectively. In the former case, the liquid-vapor interface is an adiabatic boundary and the system is the most instable; in the later case, the liquid-vapor interface is an isothermal boundary and the system is unconditionally stable because the Marangoni convection can not be established without the temperature difference along the interface.

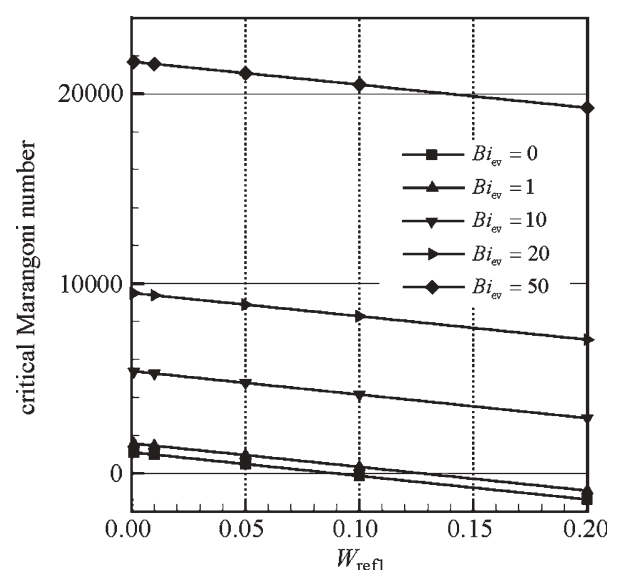

Fig. 5 Critical Marangoni number $M a_{c}$ versus evaporating velocity for different evaporation Biot numbers $(G r=0)$ 


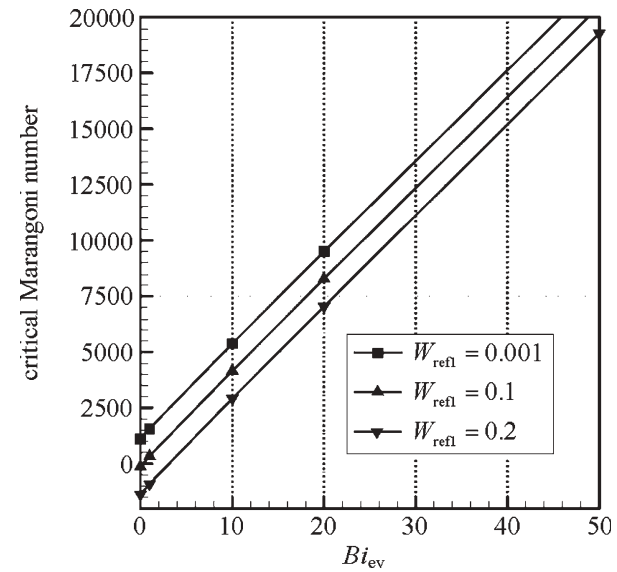

Fig. 6 Critical Marangoni number $M a_{c}$ versus evaporating Biot number $B i_{\mathrm{ev}}$ for different evaporating velocities

Figure 5 shows that the relation between the critical Marangoni number and the evaporating velocity is close to a linear one.

Figure 6 shows that the relation between the critical Marangoni number of the system and the evaporation Biot number is close to a linear one.

\subsection{Comparison between observations and experiments}

In order to validate our physical model and the analyses in the present paper, we present our numerical results in comparison with the experimental observations and results.

\subsubsection{Comparison with Block's description}

The experimental results show that the convective flow occurs in a thin evaporating liquid layer as long as the evaporation at the interface is strong enough even though the layer is cooled from below. It is taken for granted that a positive temperature gradient comes into being as long as the layer is cooled from below. In our model, "cooling from below" does not mean that the temperature at the bottom is lower than that at the interface, but means that it is lower than that of the top plate (the environment temperature). The so called "cooling" is relative to the environment.

\subsubsection{Comparison with experimental results}

In An-Ti Chai and Nengli Zhang's work [11], MarangoniBénard instability and convection in evaporating liquid layers were investigated through flow visualization and temperature profile measurement. By varying the thickness of the layer, the stability status of the evaporating liquid layer can be changed. The Marangoni numbers obtained from the measurement in the experiment are given in Table 3 for two typical cases.

In the experiment, the main source of uncertainties comes from the measurements of temperature difference and evaporation rate. The maximum uncertainty in these measurements
Table 3 Two sets of experimental cases and correspondent Marangoni number

\begin{tabular}{lllll}
\hline & $T_{\text {bottom }} /\left({ }^{\circ} \mathrm{C}\right)$ & $W_{\text {ref1 }} /\left(\mathrm{m} \cdot \mathrm{s}^{-1}\right)$ & $H_{2} / \mathrm{mm}$ & $M a_{2}$ \\
\hline Case 1: & 25 & $6.75 \times 10^{-4}$ & 1 & 351 \\
Case 2: & 25 & $6.75 \times 10^{-4}$ & 2 & 1228 \\
\hline
\end{tabular}

Table 4 Minimum and maximum possible values for $M a_{2}$

\begin{tabular}{lll}
\hline & Min & Max \\
\hline Case 1: & 430 & 645 \\
Case 2: & 982 & 1473 \\
\hline
\end{tabular}

of temperature difference is estimated to be $22.7 \%$ for alcohol. The values of $M a_{2}$ estimated by the method of single sample experiments are listed in Table 4.

In our computation, relation (52) can be used to evaluate the evaporation Biot number, however, the evaporation accommodating coefficient has not been given in the experiments. For alcohol with an accommodation coefficient $\beta=$ 1.0 , we find $B i_{\mathrm{ev}} \simeq 15.0$ with $h=1 \mathrm{~mm}$, and $B i_{\mathrm{ev}} \simeq 30.0$ with $h=2 \mathrm{~mm}$. The accommodation coefficient could be

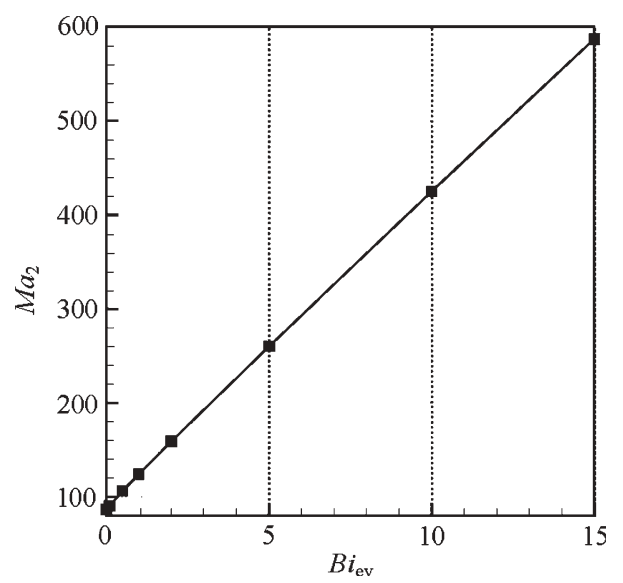

Fig. $7 M a_{2}$ versus evaporating Biot number $B i_{\mathrm{ev}}$ for the experiment Case 1

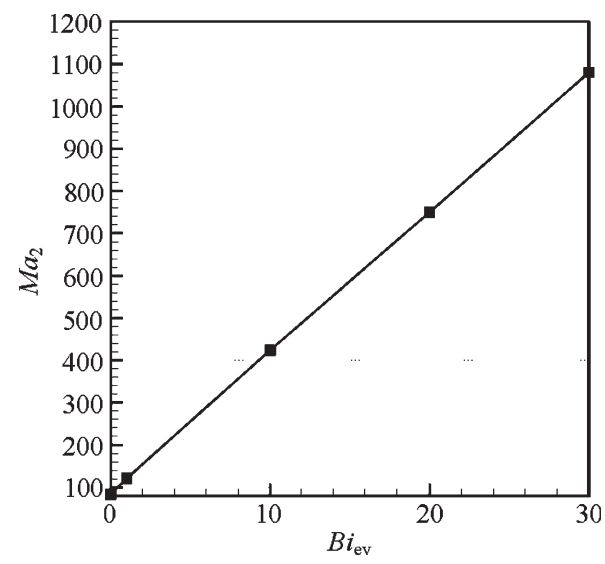

Fig. $8 M a_{2}$ versus evaporating Biot number $B i_{\mathrm{ev}}$ for the experiment Case 2 
significantly reduced by impurities at the interface, leading to a much lower value. Here we assume that the interface is not impure and in this case $\beta=1.0$. As the evaporation Biot number may vary over a wide range, plots of our computational results are extended from $B i_{\mathrm{ev}}=1.0$ to the value of $B i_{\mathrm{ev}}$ for $\beta=0.0$ in Fig. 7 and Fig. 8. The Marangoni number is seen to increase linearly with the evaporating Biot number. In Fig. 7, the evaporation velocity and depth in our model is the same as that in experiment Case 1. The Marangoni number for $\beta=1.0$ or $B i_{\mathrm{ev}}=15$ is 587 , while the Marangoni number in the experiment is between 430 and 645. In Fig. 8, the Marangoni number for $\beta=1.0$ or $B i_{\mathrm{ev}}=30$ is 1080 , and this result agrees with the experimental result in Table 4 as well.

\section{Conclusions}

A linear stability analysis for normal modes is carried out to study the onset of Marangoni-Bénard convection in a liquid layer underlying a vapor layer. Our main results can be summarized as follows. First, the temperature difference in the liquid increases with the reference evaporation velocity, and the system becomes more and more unstable. Second, the evaporation Biot number increases with the evaporation accommodation coefficient, which represents the evaporative ability, and the stability of system is enhanced with the increase of Biot number.

In summary, classical theories have only successfully provide an explanation to convection in a liquid layer heated from below without evaporation. However, these theories can not account for the convection in an evaporating thin layer, especially, with the liquid layer cooled from below. In our two-sided definite depth model, instability occurs even when the evaporating liquid layer is cooled from below. This new phenomenon could not occur in the classical one-sided model.

\section{Scope for future work}

In the present paper, we only consider the Marangoni-Bénard problem in an evaporation liquid-vapor system. There are some key issues that remain to be solved in the evaporation convection problem. In our further work, influence of the interface deformation and the vapor-recoil effect on the instability of the system will be discussed in detail.

\section{Nomenclature}

Notations

$H_{1} \quad$ depth of vapor layer

$\mathrm{H}_{2}$ depth of vapor layer

$T_{\text {top }} \quad$ the temperature at the top plate

$T_{\text {bottom }}$ the temperature at the bottom plate
$W_{\text {ref1 }}$ the base evaporation velocity of the vapor

$W_{\text {ref2 }}$ the base vertical velocity of the liquid

$J_{\text {ref }}$ the base evaporation flux of the vapor

$u_{i} \quad$ horizontal velocity

$w_{i} \quad$ vertical velocity

$p_{i} \quad$ pressure

$T_{i} \quad$ temperature

$\Delta T \quad$ temperature difference of system

$\Delta T_{2} \quad$ temperature difference in the liquid layer

$w_{\text {int }} \quad$ vertical velocity of the interface

$u_{i}^{\prime} \quad$ perturbation of horizontal velocity

$w_{i}^{\prime} \quad$ perturbation of vertical velocity

$p_{i}^{\prime} \quad$ perturbation of pressure

$T_{i}^{\prime} \quad$ perturbation of temperature

$w_{\text {int }}^{\prime} \quad$ perturbation of vertical velocity of the interface

$\tilde{U}_{i} \quad$ amplitude of $u_{i}^{\prime}$

$\tilde{W}_{i} \quad$ amplitude of $w_{i}^{\prime}$

$\tilde{P}_{i} \quad$ amplitude of $p_{i}^{\prime}$

$\tilde{\Theta}_{i} \quad$ amplitude of $T_{i}^{\prime}$

$\hat{U}_{i k} \quad$ spectral coefficient of $\tilde{U}_{i}$

$\hat{W}_{i k} \quad$ spectral coefficient of $\tilde{W}_{i}$

$\hat{P}_{i k} \quad$ spectral coefficient of $\tilde{P}_{i}$

$\hat{\Theta}_{i k} \quad$ spectral coefficient of $\tilde{\Theta}_{i}$

$g \quad$ acceleration of gravity

$p_{S}(T) \quad$ saturate evaporation pressure

$J \quad$ evaporation flux

$L \quad$ evaporation latent heat

$M \quad$ molecular weight of the vapor

$R \quad$ universal gas constant

$x \quad$ horizontal coordinate

$z \quad$ vertical coordinate

$\mathrm{Ca} \quad$ Capillary number

$\mathrm{Ga} \quad$ Galileo number

$B i \quad$ Biot number

Gr Grashof number

$\mathrm{Pr} \quad$ Prandtl number

$M a \quad$ Marangnoni number of the system

$B i_{\mathrm{ev}} \quad$ evaporation Biot number

$\mathrm{Ma}_{2} \quad$ Marangnoni number of liquid layer

E dimensionless parameter defined in Eq.(51)

Mathematical symbols

$w(x) \quad$ weight function

$\mathcal{L} \quad$ differential operator

$\mathcal{B}_{i} \quad$ differential operator of Boundary conditions

$u_{N} \quad$ truncated series

$R_{N} \quad$ residual

$\varphi_{k}(x)$ expansion function

$\hat{u}_{k} \quad$ spectral coefficient

$(,)_{w} \quad$ scalar product

$H_{w}^{p} \quad$ the weighted Sobolev space of order $p$

\|\|$_{w}^{p} \quad$ normal defined by Eq.(61)

$T_{n}(x) \quad n$-th degree Chebyshev polynomial 


$\begin{array}{ll}\mathrm{D} & \text { differential operator } \\ \nabla & \text { Laplace operator } \\ & \text { Greek symbols } \\ \nu_{i} & \text { kinetic viscocity } \\ \mu_{i} & \text { dynamic viscocity } \\ \rho_{i} & \text { density } \\ \chi_{i} & \text { thermal conductivity } \\ \beta_{i} & \text { thermal expansion coefficient } \\ \beta & \text { evaporation accommodating coefficient } \\ \lambda & \text { time growth factor } \\ \nu^{*} & \text { ratio of kinetic viscocity } \\ \mu^{*} & \text { ratio of dynamic viscocity } \\ \rho^{*} & \text { ratio of density } \\ \chi^{*} & \text { ratio of thermal conductivity } \\ \beta^{*} & \text { ratio of thermal expansion coefficient }\end{array}$

Superscripts

a perturbation quantity

\section{Subscripts}

1 a property of the vapor layer

2 a property of the liquid layer

int a property at the interface

ref a property of the base state

\section{References}

1. Bénard, H.: Les tourbillons cellulaires dans une nappe liquide. Rev Gen Sci Pures Appl 11, 1261-1271 (1900)

2. Rayleigh, L.: On convection currents in a horizontal layer of fluid, when the higher temperature is on the under side. Phil Mag 32(6), 529-546 (1916)

3. Block, M.J.: Surface tension as the cause of Bénard cells and surface tension deformation in a liquid film. Nature 178, 650-651 (1956)

4. Pearson, J.R.A.: On convection cells induced by surface tension. J Fluid Mech 4, 489-500 (1958)

5. Nield, D.A.: Surface tension as the cause of Bénard cells and surface deformation in a liquid film. J Fluid Mech 19, 341-352 (1964)

6. Palmer, H.J.: The hydrodynamic stability of rapid evaporating liquids at reduced pressure. J Fluid Mech 75, 487-511 (1976)
7. Prosperetti, A., Plesset, M.S.: The stability of an evaporating liquid surface. Phys Fluids 7(7), 1590-1602 (1984)

8. Bruelbach, J.P., Bankoff, S.G., Davis S.H.: Nonlinear stability of evaporating/condensing liquid films. J Fluid Mech 195, 463-494 (1988)

9. Colinet, P., Legros, J.C., Velarde, M.G.: Nonlinear Dynamics of Surface-Tension-Driven Instabilities. Berlin: Wiley-VCH Verlag Berlin $\mathrm{GmbH}, 2001$

10. Zhang, N., Chao, D. F.: Mechanisms of convection instability in thin liquid layers induced by evaporation. Int Comm Heat Mass Transfer 26(8), 1069-1080 (1999)

11. Chai, A., Zhang, N.: Experimental study of Marangoni-Bénard convection in a liquid layer induced by evaporation. Experiment Heat Transfer 11, 187-205 (1998)

12. Bedeaux, D., Kjelstrup, S.: Transfer coefficient for evaporation. Physica A 270, 413-426 (1999)

13. Rahimi, P., Ward, C.A.: Effect of pressure on the rate of evaporation from capillaries: statistical rate theory approach. Int J Heat Mass Transfer 47, 877-886 (2004)

14. Fang G., Ward C.A.: Temperature measured close to the interface of an evaporating liquid. Physical Review E 59(1), 417-428 (1999)

15. Ward, C.A., Stanga, D.: Interfacial conditions during evaporation or condensation of water. Physical Review E 64(1), (051509-1)(051509-9) (2001)

16. Kennard, E.K.: Kinetic Theory of Gases. New York: McGraw-Hill, 1938

17. Batchlor, G.K.: An Introdution to Fluid Dynamics. Cambridge: Cambridge University Press, 1970

18. Liu, Q.S., Zhou, B.H., Nguyen, T.H., et al.: Instability of twolayer Rayleigh-Bénard convection with interfacial thermocapillary effect. Chin Phys Lett 21(4), 686-688 (2004)

19. Roget, P: Spectral Methods for Incompressible Viscous Flow, Springer, 2000

20. Orzag, S.A.: Accurate solution of the Orr-Sommerfeld stabilty equation. J Fluid Mech 50, 689-703 (1971)

21. Liu, R., Liu, Q.S., Hu, W.R.: Marangoni-Bénard instability with the exchange of evaporation at liquid-vapor interface. Chin Phys Lett 22(2), 402-405 (2005)

22. Lide, D.R., Kehiaian, H.V.: CRC Handbook of Thermophysical and Thermochemical Data. Boca Raton: DRC Press, 1994

23. Liu, Q.S.: Marangoni convection in multiple liquid layers. Acta Mechanica Sinica 34(4), 481-491 (2002) (in Chinese)

24. Liu, Q.S., Kang, Q., Hu W. R.: The effect of air layer on the Marangoni-Bénard convection of liquid layer in an enclosed cavity. J Microgravity and Space Station Utilization 3(2), 31-38 (2001) 